\title{
Continued Successful SA Recovery during the Maintenance Stage: Intra-Individual Resource Loss \& Gain Predict Relapse*
}

\author{
Lisa C. Walt, Ed Stevens, Leonard A. Jason, Joseph R. Ferrari \\ DePaul University, Chicago, USA \\ Email: lwalt@depaul.edu
}

Received December 16, 2011; revised December 29, 2011; accepted January 16, 2011

\begin{abstract}
Objectives: We combine Hobfoll's Conservation of Resources (COR) theory and key components of self-help group "step work" ideology to investigate how dynamic changes in key intra-individual resource loss and gains (self-esteem, abstinence self-efficacy, existential growth) influence relapse rates in a sample of individuals in the Maintenance Stage of substance abuse recovery. Methods: Participants $(n=579)$ completed two surveys over a nine month period that assessed baseline and changes in intra-individual loss and gain resources as well as relapse rates over study course. Multiple regression analyses were performed to predict whether baseline and dynamic changes in intra-individual scores predict relapse rates over time. Results: Individuals that reported lower levels of resource gain at baseline, as well as decreased gain trajectories and increased loss trajectories over time were more likely to relapse. Conclusions: Findings support self-help group "step work" models and the application of COR theory for relapse likelihood prediction in a sample of individuals in longer term substance abuse recovery. Research efforts should examine the complex relationship between these dynamic intra-individual resources, social cognition, self-regulation and relapse risk. Future interventions should address the importance of the continual development and protection of these valuable intra-individual resources to prevent relapse.
\end{abstract}

Keywords: Substance Abuse; Core; Maintenance

\section{Introduction}

Substance Use Disorders (SUDs) affect approximately $10 \%$ of the population, and have been associated with several negative social, mental, and physical health outcomes including relationship dysfunction, employment loss, and death [1]. Recently, researchers have conceptualized SUDs as chronic illnesses that may require cyclical and lifelong treatment, based on individuals' changing needs, risks, and stressful life events [2-5]. Thus, it is important to add to current substance abuse (SA) research findings to determine what unique factors may protect individuals against relapse during life course.

The term "recovery" originated from self-help programming and "step work". "Step Work" was predicated on the belief that individuals should progress through a series of dynamic changes that stem from the acknowledgment of responsibility for one's actions, acceptance of a higher power, and the building of their own existential growth

\footnotetext{
${ }^{*}$ The authors appreciate the financial support from the National Institute on Drug Abuse (NIDA grant numbers DA13231 and DA19935), and the National Center on Minority Health and Health Disparities (grant MD002748).
}

and understanding. Furthermore, SA self-help literature emphasized that this engagement in "step work" or "working a program" would produce better longer term SA recovery outcomes [6-8].

Historically, addiction researchers have struggled to agree on a definition of successful SA "recovery" [9]. However, the gradual shift in the etiological understanding of SUDs (from character "flaw" to chronic illness), as well as the purported effectiveness of self-help programs, has stimulated a renewed interest in the operationalization and measurement of the concept of "recovery" [9]. Most recently, the Substance Abuse and Mental Health Services Administration's Recovery Support Strategic Initiative [10] developed a working definition of recovery, which defined recovery as "a process of change through which individuals work to improve their own health and wellbeing, live a self-directed life, and strive to achieve their own full potential". This definition highlights the intraindividual and growth processes (e.g., increases in selfesteem, abstinence specific self-efficacy, existential growth) that seem to be important for lifetime SA recovery.

The current project attempts to combine key compo- 
nents of Hobfoll's Conservation of Resources Theory (COR) [11] to explore the dynamic relationship between three intra-individual resources (self-esteem, abstinence self-efficacy, potential for existential growth) and relapse risk in a sample of individuals that have been in recovery for more than 6 months. In order to accomplish this task, we will first describe relevant literature and theory that links certain "recovery resources" (self-esteem, abstinence selfefficacy, existential growth) to successful SA recovery initiation and maintenance. Next, we will use Hobfoll's [11] Conservation of Resources (COR) theory to explain how dynamic changes in these "recovery resources" may influence relapse risk for those in more "stable" recovery (categorized using the Transtheoretical Model of Health Behavior Change-Maintenance Stage). Finally, we will empirically test the dynamic relationship between loss and gain of these intra-individual resources and one-year relapse rates in our sample.

Many researchers have suggested that substance abuse recovery, like other healthy behavior change attempts, may follow a cyclical and progressive stage pattern [12]. Furthermore, research has also illustrated that interventions aimed at changing health behaviors may be more effective if they are tailored to address the relevant challenges, needs, emotions, and cognitions present at each stage of behavioral change (see the Transtheoretical Model of Health Behavior Change; TTM for a review of theoretical constructs) $[12,13]$. A person's stage is determined by two things; their intentions to engage in a target behavior, and, if applicable, the amount of time they have been engaging in this target behavior [12].

Stage models of health behavior change have been used to conceptualize the link between the factors, conditions, or "resources" that encourage individuals to first atempt drug and alcohol abstinence (TTM's Action Stage). This research has indicated that the presence of several "recovery resources" including familial and financial support, self-esteem, abstinence self-efficacy, perceptions of happiness, life satisfaction and positive coping strategies predict initial SA recovery engagement [2,5,14-16]. Recent research has also investigated how these same resources may be important during longer term recovery maintenance (6 months through several years of abstinence). Investigators have recently focused on the effects of a cluster of intra-individually derived resources (e.g., self-esteem, abstinence self-efficacy, indicators of existential growth potential) on longer term recovery trajectories [14-16]. The results from these studies combined with the SA recovery community's newer conceptualization of recovery have led us to investigate how dynamic changes in intra-individually derived resources of self-esteem, abstinence self-efficacy, and existential growth influence longer term recovery success.

Research on self-esteem (perceptions of global feelings of self-worth) and abstinence self-efficacy (one's feelings of confidence that they can avoid substance abuse across a series of drug related situations) has been linked to successful abstinence at both the Action stage (initial engagement in abstinence through six months) as well as the Maintenance stage of recovery. In fact, many SA intervention programs contain modules that specifically aim to increase these two intra-individual resources [17-19].

Existential growth (a type of introspective development that relies on an emphasis of the subjective life experience, and stems from the understanding of one's own life meaning), and the potential for existential growth has also been linked to both SA initiation (TTM Action) and continued sobriety (TTM Maintenance). Furthermore, some experts argue that SUDs may originate as a faulty self-medication coping mechanism for existential pain [20-22]. Researchers have operationalized and measured existential growth in several ways; such as a development process [23], spirituality or spiritual well-being [24,25], spiritual transcendence $[9,26]$, purpose in life or life meaning $[21,27,28]$, and life satisfaction [15].

These findings are promising, however, studies have only measured intra-individual resources at one time point; as either a predictor or outcome of relapse risk $[3,12,19]$. Thus, research has not captured the dynamic and fluctuating nature of these intra-individual resources; and how these fluctuations influence continued longer-term SA recovery success. Hobfoll's COR theory [11] may provide the dynamic mechanism in which to explain how perceived changes in key intra-individual resources (self-esteem, abstinence self-efficacy, potential for existential growth) influence relapse risk over time. COR theory is a motivational stress theory that incorporates both individual's objective and perceived environment in response to stress, and explains how stress directly and indirectly influences health and health behaviors [11]. COR theory assumes that people strive to obtain, retain, protect, and nurture resources, and what is threatening to them is the potential or actual loss of these valued resources. Thus, individuals experience psychological stress when there is an actual or perceived a) threat of a net loss of resources b) net loss of resources or c) a lack of resource gain following the investment of resources. Resources are defined as those objects, personal characteristics, conditions, or energies that are valued by the individual or that serve as a means for attainment of these objects, personal characteristics, conditions or energies [11].

COR theory also assumes that individuals must operate on two levels; one in which they consistently evaluate their risk of loss for valuable resources, and attempt to conserve these valuable resources; and another, where individuals attempt to build resource reserves. Thus, within COR theory, resource loss and gain are separate concepts, and individuals evaluate both dimensions simultaneously 
for each resource. Furthermore, resource loss may or may not influence gain, and one cannot assume that loss encounters will automatically stifle resource gain accumulation. In fact, some individual's gains come through extreme loss - such as growth through trauma or hardship.

We propose that COR theory provides a mechanism to explain how the evaluation of key intra-individual resources (self-esteem, abstinence specific self-efficacy, potential for existential growth) may predict relapse in a sample of individuals in the Maintenance Stage of SA recovery. Thus, we suggest that for those individuals that encounter intra-individual resource loss, or failure to achieve expected intra-individual resource gains over a period of one year, will suffer emotional, motivational and cognitive blows that may alter ingrained abstinent specific behaviors and consequently, lead to relapse.

Specifically, we hypothesize that 1) baseline levels of intra-individual resource gains (self-esteem, abstinence specific self-efficacy, and potential for existential growth) will predict continued SA recovery in our sample. We also hypothesize that 2) individuals reporting greater gain trajectories (negative difference scores from administration 1 to administration 2), will be less likely to relapse during the study period. Finally, we hypothesize that 3 ) individuals with higher loss trajectories (negative differrence scores from administration 1 to administration 2), will be more likely to relapse over the study period.

\section{Method}

\subsection{Participants}

Participants were adults in SA recovery who, at study inception, were residents of several different Oxford Houses across the United States. Oxford Houses are drug-free, sober housing for individuals in recovery; and residence in these homes has been positively related to recovery success (as compared to other living environments) [1, 29]. Participants in this study were also part of a large, one-year longitudinal project (see [30] for more details), where individuals were enrolled and interviewed 4 times over a one year period (every three months). Participants were recruited via direct solicitation at individual Oxford Houses, as well as the Oxford House World Services annual convention. Previous analysis did not yield significant differences between these two recruitment methods. Participants provided informed consent and were compensated $\$ 15$ for each survey battery completed.

Of the 897 initial participants in the study, a total of 579 were included in our analysis. The relevant measures were administered at two points during data collection, at Waves 1 and 3 (baseline and 9 months later); thus the 579 participants included in this investigation were participants that completed the relevant measures at baseline and nine months. An investigation of relevant missing values found that initial COR values were not significantly different between individuals that dropped out versus those who continued participation. However, length of sobriety was significantly different $\left(t_{(191)}=3.6, p=0.000\right)$; where those individuals who maintained participation in the study, on average, were sober or drug free for 7.5 months longer than those who dropped out. Thus, length of sobriety was used as a control variable in the present study, as it is predictive of relapse likelihood.

The majority of the participants were male (69.3\%), with an average age of 39.4 years $(S D=18.4$ years). Most $(58.2 \%)$ were Non-Hispanic Caucasian, with AfricanAmericans comprising the next largest racial/ethnic group (34.0\%). Both the Hispanic/Latino (3.6\%) and other (4.1\%) ethnic categories were relatively less well represented. Few of the participants were married $(3.8 \%)$, and most reported that they had never been married (52.0\%). All participants were classified as Maintenance Stage participants (mean number of years abstinent $=1.72 ; S D=$ 1.96 years, $M d=1.09$ years).

\subsection{Measures}

\subsubsection{Addiction Severity Index}

The Addiction Severity Index-lite (ASI) is an instrument that evaluates important characteristics of an individual's situation that may be associated with substance misuse, and assesses: medical and employment/support statuses, drug and alcohol use, illegal behaviors, family and social relationships, and psychiatric condition [31]. This instrument is commonly used in both research and clinical practice and is regarded as valid and reliable $(\alpha \geq 0.80$; $[31,32]$. Sociodemographic and prior length of abstinence information was obtained from this measure.

\subsubsection{Alcohol and Substance Use}

A modified version of Miller and Del Boca's [33] Form 90 Timeline Follow-back was administered at every interview, and measured drug and alcohol use in the past 90 days. The Form 90 has been found to have good reliability for the key summary measures of alcohol use and moderate reliability for commonly used drugs. Test-retest interviews have been reported as generally being consistent [33]. This information was used to capture relapse rates during the study; participants that reported any use during the study were coded as "relapsers", and those that reported no use during the study were coded as "continued abstainers". We realize that other addiction research has found the Harm Reduction Model to be useful in predicting successful outcomes; however, since we have used the Self-Help Model of Substance Abuse Recovery to inform our conceptualization of recovery, we have chosen to measure "recovery" as continued abstinence rather than reduced use. 


\subsubsection{Conservation of Resources}

A 45-item, modified version of the Conservation of Resources (COR) [11] instrument was administered at baseline and at the third interview (approximately one year apart). COR uses two identical measures, separately administered, to evaluate resource loss and resource gain. At each administration, participants were instructed to think back over the past three months, and to consider the degree to which they lost (or gained) each of 45 different resources. Each item was scored on a 5 point Likert scale that ranged from $(0=$ not at all, to $4=$ greatly $)$, and measured resource loss and gain across several domains. The present study utilized the Intra-Individual Resources subscale exclusively, an 18-item subscale that investigated personal resources drawn from expressions of global self-esteem, substance abuse self-efficacy, and existential growth potential. Example items for this subscale included "feeling that I am successful", "sense of optimism", "hope", and "knowing where I am going with my life". Internal consistency was excellent for both gains (Cronbach's alpha, $\alpha=0.970)$ and losses $(\alpha=0.964)$.

Two administrations of each COR intra-individual resource subscale (losses and gains), approximately nine months apart, resulted in 4 separate scores: initial level of COR intra-individual resource gains, initial level of COR intra-individual resource losses, the change in COR intraindividual resource loss across the two administrations (calculated by subtracting the first administration and the second administration of loss scores), and the change in intra-individual resource gain from administration 1 to 2 (calculated by subtracting the first administration and the second administration of gain scores). Negative loss change scores indicated an increase in resource losses over study duration, whereas positive change scores indicated either a stabilization or reduction of losses. Conversely, positive gain change scores indicated a decrease in gains as compared to baseline (administration 1). Negative change scores indicated a continued forward trajectory and forward momentum. Furthermore, higher negative change scores indicated a steeper gain trajectory. It is important to note that lower gain change scores do not indicate an increase in losses, but rather, indicate a faiflure to continue building intra-individual resource gains across time, resulting in a lowered gain trajectory.

\section{Results}

Approximately $78 \%$ of the sample remained abstinent over study duration. In line with Self-Help ideology, continued abstinence was defined as total abstinence for all substances including alcohol and illegal drugs. In addition, participants reported higher gains than losses at baseline (first administration; loss $M=17.33, S D=16.36$; gain $M=43.93, S D=18.04)$, as well as second administration (approximately one year later; loss $M=12.08$,
$S D=14.33$; gain $M=41.68, S D=19.67)$; participants also reported an average $2.20(M d=1.00, S D=25.78)$ decrease in gains from administration 1 to 2 , and an average $5.27(M d=3.00, S D=21.97)$ reduction in losses from administration 1 to 2 . Thus, approximately $45 \%$ of the sample reported continued forward momentum in gain accumulation trajectory, $3 \%$ of the sample reported stabilization in gain accumulation trajectory, and $52 \%$ of the sample reported at least some stalling in gain trajectories. Alternatively, $49 \%$ of the sample reported an increase in personal losses, $6 \%$ of the sample reported stabilization in loss, and $45 \%$ reported a decrease in personal losses. None of these scores (initial loss or gain, change score loss or gain) were significantly different across gender or ethnic groups $(p>0.05)$.

A multiple logistic regression was conducted using SPSS version 18, to predict participant's likelihood to relapse (yes/no) during the study duration. Four separate predicttors were simultaneously entered into the equation: total years abstinent before study enrollment, gains at baseline, change in gains from administration 1 to 2 , and change in losses from administration 1 to 2 . A test of the full model against the constant only model was statistically significant, indicating that the predictors, as a set, reliably distinguished between relapsers and continued abstainers in this sample $\left(\chi^{2}=99.04, p<0.000, d f=4\right)$. Prediction success overall was $81 \%$ (26\% correctly predicted in relapse category and $96 \%$ correctly predicted in continued abstainer category) with a Nagelkerke's $\mathrm{R}^{2}$ of 0.24 . Furthermore, each predictor entered into the equation significantly added to the model (See Table 1).

The covariate, years abstinent before study enrollment, was strongly predictive of substance use during the study $[\mathrm{OR}=0.46, \mathrm{CI}=0.37-0.57]$. Furthermore, Hypothesis 1 was supported, in that the initial level of intra-individual COR gains predicted of substance usage where individuals that reported higher levels of intra-individual gains at baseline were significantly less likely to relapse over study course $[\mathrm{OR}=0.98, \mathrm{CI}=0.96-0.99]$. For

Table 1. Personal conservation of resource scores as predictors of alcohol/drug usage.

\begin{tabular}{lccccc}
\hline Variable & B & SE & Wald & Significance Odds Ratio \\
\cline { 1 - 3 } Length of Sobriety & -0.770 & 0.108 & 50.511 & 0.000 & 0.463 \\
\cline { 3 - 3 } COR Gains-Base & -0.426 & 0.144 & 8.74 & 0.003 & 0.653 \\
Change in Gains & -0.376 & 0.113 & 11.065 & 0.001 & 0.686 \\
Change in Losses & 0.351 & 0.106 & 10.944 & 0.001 & 1.420 \\
Constant & & & & & \\
\hline
\end{tabular}


each additional point on the intra-individual gain scale, the odds of the participant being in the "relapsed" group decreased by $2 \%$. Thus, a one standard deviation increase in COR intra-individual gains decreased the odds of being in the "relapsed" group by $36 \%$.

Hypothesis 2 was also supported, in that the change in intra-individual gains from administration 1 to 2 predicted relapse. Specifically, participants that reported steady increases of COR personal gains, as evidenced by higher COR gain change scores at administration 2 (resulting in negative summed scores and beta coefficients), were less likely to relapse than persons that reported a reduction in their personal gain trajectory $[\mathrm{OR}=1.02, \mathrm{CI}=1.01-$ 1.03]. Finally, Hypothesis 3 was supported, in that change in COR intra-individual resource losses predicted relapse. Specifically, individuals that stabilized or decreased their losses, as evidenced by lower intra-individual loss scores at second survey administration (resulting in positive summed scores and beta coefficients), were significantly less likely to relapse over study course, [OR = $0.98, \mathrm{CI}=0.97-0.99]$. Note that the beta coefficient for this predictor is negative, which may seem counterintuitive. However, change in losses variable reflects the amount of change in losses from the first survey administration to the second survey administration. Thus, positive scores indicate that a participant reported fewer losses as time went on. These findings suggest that higher levels of COR intra-individual gains at study inception, continued intra-individual gain trajectories, and either stabilization or discontinued intra-individual loss trajectories over study duration best predicted relapse vulnerability in a sample of individuals in longer term recovery.

\section{Discussion}

As a whole, participants enrolled in the project were successful in their continued recovery, since approximately $77 \%$ of the sample remained abstinent for both alcohol and illegal drugs, over study duration. However, nearly one quarter of our sample were unsuccessful in their attempts at continued abstinence, which provides support for the disease model of addiction, where individuals with this disorder are believed to require either continual or periodic intervention.

Results supported all three hypotheses, suggesting that initial levels of intra-individual resource gain, as well as relative changes in the gains and losses of intra-individual resources, predicted likelihood of relapse across a one year period. This study built on previous work using the TTM [12] and COR Theory [11] to test how subjective, dynamic changes in these intra-individual resources (selfesteem, abstinence self-efficacy, potential for existential growth) may place certain individuals at-risk for relapse. The authors suggest that these intra-individual resources may be especially important for longer term recovery; as there seems to be a greater emphasis on the more "introspective" components of continued lifetime abstinence. The recent release of SAMSHAs [10] formal definition of "recovery" reflects this emphasis on introspective skill building. COR theory assumes that people attempt to maintain, garner and protect resources; and what is threatening is the perceived loss of resources, or the failure to gain resources when other resources are used to invest in further resource development. This assumption of COR may be especially important for individuals in longer term recovery, and may provide an explanation as to why some individuals relapse after several years of successful abstinence. Simply stated, expectations of continued intraindividual resources gains, in particular potential for existential growth, may be an important buffer against life's stressors and setbacks.

Individuals in Recovery Maintenance have been reasonably successful in their recovery endeavors; usually reporting at least six months of abstinence during which time most have managed to recapture at least a few of the resources they may have lost during their active addictions (e.g., employment, social supports, legal parental status). This successful abstinent time and intra-individual resource accumulation may translate into higher levels of self-esteem and abstinence self-efficacy. However, similar to self-help ideologies, we suggest that individuals in Recovery Maintenance must continue to guard themselves against assaults on their self-esteem and self-efficacy resources, while attempting to invest and accumulate existential growth resources. This process of resource protection and garnering is especially important because efforts at investment and growth are hazardous. Since COR asserts that each individual perceives their own levels of relative loss and gain in personal resources, and individuals most often carry a perception that the longer they are in recovery, the more stable their own life should become, failure to "move forward" or achieve success after investment of key personal resources may prove especially disheartening, and may lead to behaviors that are not conducive to continued drug and alcohol cessation. Failure to achieve expected gains in intra-individual resources could be the trigger for relapse; however, successful garnering and maintenance of these key resources could catalyze growth in several other areas that can provide a buffer against relapse.

Previous research has only investigated these three resources statically, and has not considered the bi-directional relationship between ongoing life stressors and levels of these intra-individual resources. We propose that the mechanism behind individual's lifelong abstinence success is this continued ability to protect and garner intra-individual resource reserves. These key resources can then be used to bolster continued motivation and perseverance in SA recovery, and may result in more recov- 
ery-conducive behaviors (e.g. actively avoiding triggers, seeking out abstinence specific support during periods of extreme stress). These findings fit well within SA self-help literature, as members discuss the importance of "working a recovery program." This "working" embodies not only successful abstinence, but also effortful exercises of introspection and growth. This final "step" in recovery is so pivotal that when an individual relapses, many other selfhelp members suggest that the relapsed individual failed to focus on their recovery, and failed to "work" at it [6].

These findings have some powerful implications for inpatient SA treatment programs as well as SA aftercare programs, and other formal self-help mentorship programs. New interventions, delivered at the inpatient and formal after-care level, could teach individuals how to manage the negative emotions that are encountered when one encounters daily stressors and setbacks, and could create opportunities for those in longer term recovery to reflect on their own personal growth and how the activities that they have chosen to engage in could help or hinder their own chances for successful lifetime recovery.

The project is not without limitations. For example, we gathered most of our data from only one type of sober living environment, the Oxford House. In addition, most of our participants were male, approximately of the same age, and had been in recovery for approximately the same amount of time (one year). Another possible limitation is the high attrition rates for our sample (retention rate $\sim 65 \%$ ). However, community research conducted with this sample is unique as compared to other types of community samples as this sample includes high-risk, urban, former substance abusers, many with criminal backgrounds. These population characteristics may contribute to increased tracking difficulties and higher attrition rates. Future studies should include larger and more diverse samples with greater variability in longer term recovery (aiming anywhere from one year of continuous recovery through several decades of successful recovery).

Our model was also a simple model that investigated the additive effects of intra-individual resource baseline gains, change in intra-individual resource gains, and change in intra-individual resource losses. Future research should develop more complicated and integrative models that illustrate the dynamic effect of intra-individual resource loss and gain. Furthermore, since researchers have already established that key resources predict SA treatment success and early term recovery; researchers might also attempt to understand how intra-individual resource loss and gain might influence the loss and gain of other important resources, and how this relationship influences recovery across life course. In addition, researchers might want to understand how other social cognitive and emotive factors influence the pathway between the experience of daily stressors and setbacks, and their resulting influence on these intra-individual resources.

\section{REFERENCES}

[1] L. A. Jason, B. D. Olson and K. Foli, "Rescued Lives: The Oxford House Approach to Substance Abuse," Routledge, New York, 2008.

[2] W. Cloud and R. Granfield, "The Social Process of Exiting Addiction: A Life Course Perspective," Nordic Studies on Alcohol and Drugs, Vol. 44, 2004, pp. 185-202.

[3] M. L. Dennis, M. A. Foss and C. K. Scott, "An EightYear Perspective on the Relationship between the Duration of Abstinence and Other Aspects of Recovery," Evaluation Review, Vol. 31, No. 6, 2007, pp. 585-612. doi:10.1177/0193841X07307771

[4] A. B. Laudet, J. B. Becker and W. L. White, "Don't Wanna Go through That Madness No More: Quality of Life Satisfaction as Predictor of Sustained Remission from Illicit Drug Misuse," Substance Use \& Misuse, Vol. 44, No. 2, 2009, pp. 227-252. doi:10.1080/10826080802714462

[5] R. H. Moos and B. Moos, "Protective Resources and LongTerm Recovery from Alcohol Abuse Disorders," Drug and Alcohol Dependence, Vol. 86, No. 1, 2007, pp. 46-54. doi:10.1016/j.drugalcdep.2006.04.015

[6] Alcoholics Anonymous, "Alcoholics Anonymous," 4th Edition, A.A. World Services, New York, 2001.

[7] K. Humphreys, et al., "Self-Help Organizations for Alcohol and Drug Problems: Toward Evidence Based Practice and Policy," Journal of Substance Abuse Treatment, Vol. 26, No. 3, 2004, pp. 151-158. doi:10.1016/S0740-5472(03)00212-5

[8] H. Riper, J. Kramer, F. Smit, B. Conijn, G. Schippers and P. Cuipers, "Web-Based Self-Help for Problem Drinkers: A Pragmatic Randomized Trial," Addiction, Vol. 103, No. 2, 2007, pp. 218-227. doi:10.1111/j.1360-0443.2007.02063.x

[9] A. B. Laudet, "What DOes Recovery Mean to You? Lessons from the Recovery Experience for Research \& Practice," Journal of Substance Abuse Treatment, Vol. 33, No. 3, 2007, pp. 243-256. doi:10.1016/j.jsat.2007.04.014

[10] Substance Abuse and Mental Health Services Administration (SAMSHA), "Working Definition of Recovery," 2011.

http://blog.samhsa.gov/2011/08/12/recovery-defined-\%E $2 \% 80 \% 93$-give-us-your-feedback

[11] S. E. Hobfoll, "Conservation of Resources: A New Attempt at Conceptualizing Stress," American Psychologist, Vol. 44, No., 1998, pp. 513-524. doi:10.1037/0003-066X.44.3.513

[12] DiClemente, "Schumann, Green \& Early," 2011.

[13] J. O. Prochaska and C. C. DiClemente, "Stages and Processes of Self-Change of Smoking: Toward an Integrative Model of Change. Journal of Consulting \& Clinical Psychology, Vol. 51, No. 3, 1983, pp. 390-395. doi:10.1037/0022-006X.51.3.390

[14] W. Cloud and R. Granfield, "Conceptualizing Recovery Capital: Expansion of a Theoretical Construct," Substance 
Use \& Misuse, Vol. 43, No. 12-13, 2008, pp. 1971-1986. doi:10.1080/10826080802289762

[15] A. B. Laudet and W. L. White, "Recovery Capital as Prospective Predictor of Sustained REcovery, Life Satisfaction, and Stress among Former Poly-Substance Abusers," Substance Use \& Misuse, Vol. 43, No. 1, 2008, pp. 27-54.

[16] R. Moos, "Theory-Based Active Ingredients of effective Treatments for Substance Use Disorders," Drug Alcohol Dependence, Vol. 88, No. 2-3, 2007, pp. 109-121. doi:10.1016/j.drugalcdep.2006.10.010

[17] K. S. Lam, "Life beyond Sobriety: A Developmental Framework to Restore Normal Development during Recovery from Substance Addiction," Journal of Family Psychotherapy, Vol. 21, No. 4, 2010, pp. 299-304. doi: 10.1080/08975353.2010.529409

[18] J. A. Stien, E. L. Dixon and A. M. Nyamathi, "Effects of Psychosocial and Situational Variables on Substance Abuse among Homeless Adults," Psychology of Addictive Behaviors, Vol. 22, No. 3, 2008, pp. 410-416. doi:10.1037/0893-164X.22.3.410

[19] E. Da S. Cardoso, C. C. Chou, N. Y. Yasui, J. Chronister, F. Chan, V. Lyons and V. R. Muller, "The Effect of Task Specific Self-Efficacy on Stages of Change of People with Substance Abuse Problems," Australian Journal of Rehabilitation Counseling, Vol. 15, No. 2, 2009, pp. 6373. doi:10.1375/jrc.15.2.63

[20] S. Ventegodt, J. Merrick and N. J. Andersen, "Quality of Life as Medicine. II. A Pilot Study of Five Day 'Quality of Life \& Health' Cure for Patients with Alcoholism," The Scientific World Journal, Vol. 3, 2003, pp. 842-852. doi:10.1100/tsw.2003.67

[21] A. B. Laudet, K. Morgen and W. L. White, "The Role of Social Supports, Spirituality, Religiousness, Life Meaning and Affiliation with 12-Step Fellowships in Quality of Life Satisfaction among Individuals in Recovery from Alcohol and Drug Problems," Alcohol Treatment Quarterly, Vol. 24, No. 5-6, 2006, pp. 33-73. doi:10.1300/J020v24n01 04

[22] L. Wiklund, "Patient Perspectives: Existential Aspects of Living with Addiction-Part I: Meeting Challenges. Journal of Clinical Nursing Vol. 17, No. 18, 2008, pp. 24262434. doi:10.1111/i.1365-2702.2008.02356.x

[23] K. E. Hart and T. Singh, "An Existential Model of Flourishing Subsequent to Addiction: The Importance of Living a Meaningful and Spiritual Life," Illness, Crisis \& Loss, Vol. 17, No. 2, 2009, pp. 125-147.
doi:10.2190/IL.17.2.d

[24] G. Chen, "Social Support, Spiritual Program and Addiction Recovery," International Journal of Offender Therapy and Comparative Criminology, Vol. 50, No. 3, 2006, pp. 306-323. doi:10.1177/0306624X05279038

[25] J. Lillis, E. Gifford, K. Humphreys and R. Moos, “Assessing Spirituality/Religiosity in the Treatment Environment: The Treatment Spirituality/Religiosity Scale," Journal of Substance Abuse Treatment, Vol. 35, No. 4, 2008, pp. 427-433. doi:10.1016/j.jsat.2008.02.002

[26] R. L. Piedmont, "Spiritual Transcendence as a Predictor of Psychosocial Outcome from an Outpatient Substance Abuse Program," Psychology of Addictive Behaviors, Vol. 18, No. 3, 2004, pp. 213-222. doi:10.1037/0893-164X.18.3.213

[27] G. C. B. Lyons, F. P. Deane and P. J. Kelly, "Forgiveness and Purpose in Life as Spiritual Mechanisms of Recovery from Substance Use Disorders," Addiction Research \& Theory, Vol. 18, No. 5, 2010, pp. 528-543. doi: $10.3109 / 16066351003660619$

[28] R. A. Martin, S. MacKinnon, J. Johnson and D. J. Rohsenow, "Purpose in Life Predicts Treatment Outcome among Adult COcaine Abusers in Treatment," Journal of Substance Abuse Treatment, Vol. 40, No. 2, 2011, pp. 183188. doi:10.1016/j.jsat.2010.10.002

[29] L. A. Jason, M. I. Davis and J. R. Ferrari, "The Need for Substance-Abuse Aftercare: Longitudinal Analysis of Oxford House," Addictive Behaviors, Vol. 32, No. 4, 2007, pp. 803-818. doi:10.1016/j.addbeh.2006.06.014

[30] A. T.McLellan, H. Kusher, D. Metzger, R. Peters, I. Smith, G. Grissom and M. Argerion, "The Fifth Edition of the Addiction Severity Index," Journal of Substance Abuse Treatment, Vol. 9, No. 3, 1995, pp. 199-213.

[31] J. S. Cacciola, A. I. Alterman, A. T. McLellan, Y.-T. Lin and K. G. Lynch, "Initial Evidence for the Reliability and Validity of a 'Lite' Version of the Addiction Severity Index," Drug and Alcohol Dependence Vol. 87, No. 2-3, 2007, pp. 297-302. doi:10.1016/j.drugalcdep.2006.09.002

[32] W. R. Miller and F. K. Del Boca, "Measurement of Drinking Behavior Using the form 90 Family of Instruments," Journal of Studies on Alcohol Supplement, Vol. 12, 1994, pp. 112-118.

[33] J. S. Tonigan, W. R. Miller and J. M. Brown, "The Reliability of Form 90: An Instrument for Assessing Alcohol Treatment Outcome," Journal of Studies on Alcohol, Vol. 58, No. 4, 1997, pp. 358-364. 